\title{
ROBUST ESTIMATION OF STANDARD DEVIATION
}

\author{
G.U. Fenstad, M. Kuernes and L. Walløe \\ UNIVERSITY OF OSLO
}

Abstract. Six different estimators of standard deviation have been compared by stochastic simulations and by asymptotic calculations. The observations were independent, identically distributed either with a normal distribution or with a distribution in the "neighbourhood" of a normal distribution.

The simulations showed that the usual standard deviation estimator is too sensitive against deviations from normality. Estimators based on the absolute deviation or on a fractile-difference turned out to be better under the considered deviations from normality, and compared well with the usual estimator under normal assumptions.

The asymptotic calculations showed that comparing asymptotic values of two estimators may give a false impression of their corresponding finite properties.

Key words: robust estimation, standard deviation, stochastic simulation. 


\section{INTRODUCTION}

On the basis of independent, identically distributed observations $x_{1}, \ldots, x_{n}$ we want to estimate their common standard deviation (SD).

If the observations are normally distributed the minimum variance unbiased estimator is:

$$
s_{1}=k_{1}(n)\left(\sum_{i=1}^{n}\left(x_{i}-\bar{X}\right)^{2} /(n-1)\right)^{\frac{1}{2}}
$$

where $\bar{X}$ is the arithmetic mean and $k_{1}(n)$ is a correction factor for biasedness depending on $n$. If, however, the assumption of normality is violated, we do not know much about the finite properties of $S_{1}$ relative to the properties of other estimators.

Bickel and Lehmann (1976) have studied asymptotic relative efficiencies of different estimators for dispersion under nonnormal assumptions.

In this paper we have compared different SD-estimators for $\mathrm{n}$ finite as well as infinite, when the distributions of the observations are in the "neighbourhood" of the normal distribution.

\section{ChOICE OF DENSITIES}

Our choices of the common distribution function and density function, $F$ and $f$ respectively, are motivated by our wish to study the following two situations:

A The shape of the density $f$ differs slightly from the normal density $(\xi, \sigma)$. However, the expectation and SD in $f$ is still $\xi$ and $\sigma$, respectively.

B The density of the underlying distribution of the sample is normal $(\xi, \sigma)$, but the set of observations may contain 
small proportion of wild observations with SD larger than $\sigma$.

In situation A we want to estimate the common SD of all the observations in the sample, while we in situation B want to estimate the SD of the non-wild observations.

To examplify situation A we have chosen to study the following cases:

(i) $f$ is the density of $a V+b$ where $V$ is $t$-distributed with $v$ degrees of freedom, $v=5,6, \ldots, 10$.

(ii) $f$ is the density of $a W+b$ where $w$ is $x^{2}$-distributed with $v$ degrees of freedom, $v=3,4, \ldots, 10$.

The constants a and $\mathrm{D}$ are for each density chosen such that expectation and SD are $\xi$ and $\sigma$, respectively.

The densities in case (i) are for large $v$ very similar to the normal density $(\xi, \sigma)$, but their tails are heavier. In case ( $i i)$ the densities also are similar to the normal density $(\xi, \sigma)$ for large $v$, but they are skewed to the right. We have also considered densities with tails lighter than the normal density. Some of the densities in case (i) and (ii) compared to the normal density $(\xi, \sigma)$ are shown in Figure $1(a)-(c)$.

To examplify situation $B$ we have chosen to study the case:

(iii) $f$ is given by

$$
f(x)=(1-\varepsilon) \frac{1}{\sigma} \varphi\left(\frac{x-\xi}{\sigma}\right)+\varepsilon \frac{1}{3 \sigma} \varphi\left(\frac{x-\xi}{3 \sigma}\right)
$$

where $\varphi$ is the normal density $(0,1)$. We have considered $\varepsilon$ in the interval $[0,0.10]$.

In Figure 1(d) the density $f$ with $\varepsilon=0.05$ is compared with the normal density $(\xi, \sigma)$. 


\section{ESTIMATORS}

We have compared six SD-estimators. They are based on the following estimators being asymptotic unbiased under normal assumptions:

$$
\begin{aligned}
& T_{1}=\left(\sum_{i=1}^{n}\left(X_{i}-\bar{X}\right)^{2} /(n-1)\right)^{\frac{1}{2}} \\
& T_{2}=\sqrt{\pi / 2} \cdot \sum_{i=1}^{n}\left|X_{i}-\bar{X}\right| / n \\
& T_{3}=" 10 \% \text { upper trimmed mean of }\left|x_{i}-\hat{\mu}_{0.50}\right| " / c_{0.10} \\
& \mathrm{~T}_{4}=\left(\hat{\mu}_{0.841}-\hat{\mu}_{0.259}\right) / 2 \cdot u_{0.801} \\
& T_{5}=\left(\hat{\mu}_{0.75}-\hat{\mu}_{0.25}\right) / 2 \cdot L_{0.75} \\
& \mathrm{~T}_{6}=\operatorname{median}\left\{\left|X_{i}-\hat{\mu}_{0.50}\right| j / u_{0.75}\right.
\end{aligned}
$$

where $u_{p}$ is the p-fractile in the standard normal distribution, $\hat{\mu}_{\mathrm{p}}$ is the empirical p-fractile in the sample (linear interpolation when necessary) and $c_{\alpha}=2\left[\varphi(0)-\varphi\left(u_{1}-\alpha / 2\right)\right] /(1-\alpha)$ $\left(c_{0.10}=0.6573\right)$.

The estimators $T_{i}, i=1, \ldots, 6$ are biased for finite $n$. Therefore we introduce a correction factor $k_{i}(n)$, such that $S_{i}=k_{i}(n) T_{i}$, $i=1, \ldots, 6$ are unbiased or approximately unbiased under normal assumptions, $k_{i}(n) \rightarrow 1$ as $n \rightarrow \infty$.

For $i=1$ and 2 there are explicit expressions for $k_{i}(n)$. For $i=4$ and 5 we have fitted an exponential curve to $\mathrm{ET}_{i}$, $n=15, \ldots, 50$ (computed by using tables of expected values of 
order statistics from the normal distribution) by least squares. For $i=3$ and 6 we have fitted exponential curves to simulated estimates of $E T_{i}, n=5, \ldots, 50$. Thus $k_{i}(n), i=3,4,5,6$ are the inverse of the fitted curves:

$$
\begin{aligned}
& k_{1}(n)=\sqrt{\frac{n-1}{2}} \frac{\Gamma\left(\frac{n-1}{2}\right)}{\Gamma\left(\frac{n}{2}\right)} \\
& k_{2}(n)=\sqrt{\frac{n}{n-1}} \\
& k_{3}(n)=\left(1.0-0.1605 \cdot e^{-0.0776 \cdot n}\right)^{2} \\
& k_{4}(n)=\left\{\begin{array}{l}
\text { See Table } 1 \text { For } n<15 \\
\left(1.0-0.02464 \cdot e^{-5003475 n}\right)^{-1} \text { for } n \geq 15
\end{array}\right. \\
& k_{5}(n)=\left\{\begin{array}{l}
\text { Se Table } 1 \text { for } n<15 \\
\left(1.0-0.2107 \cdot e^{-0.03277 \cdot n}\right)^{-1} \text { for } n \geq 15
\end{array}\right. \\
& k_{6}(n)=\left(1.0-0.5868 \cdot e^{-0.3311 \cdot n}-0.08131 \cdot e^{-0.03400 \cdot n}\right)^{-1}
\end{aligned}
$$

We found it necessary to tabulate $k_{i}(n), i=4,5$ for $n<15$ because of the pronounced periodicity of $\mathrm{ET}_{i}, i=4,5$ due to the interpolation. For $n \geq 15$ the difference between the estimator using the exponential fitting and the estimator using the table values is small compared to the SD. We have used the curves in the simulations.

In Figure $2\left[k_{i}(n)\right]^{-1}$ is compared with $\mathrm{ET}_{i}$ or a simulated estimate of $\mathrm{ET}_{i}, i=1,3,5$. In Figure $2(b)\left[k_{5}(n)\right]^{-1}$ is fitted to the expected values and in Figure $2(c)\left[k_{3}(n)\right]^{-1}$ is fitted to the simulated estimates. To judge the accuracy of the fitting we also show the exact $\left[k_{1}(n)\right]^{-1}$ in Figure $2(a)$. 
$\mathrm{S}_{1}$ and $\mathrm{S}_{2}$ are probably the most used SD-estimators. $\mathrm{S}_{4}$ and $S_{5}$ are based on fractile-differences, while $S_{6}$ has been suggested by Hampel (see Andrews (1972)) as a robust SDestimator.

$\mathrm{S}_{3}$ is our suggestion of a trimmed SD-estimator. The $10 \%$ largest of $\left|x_{i}-\hat{\mu}_{050}\right|, i=1, \ldots, n$ are trimmed away before taking the mean of the left observations. We feel that this way of trimming take better care of the sken distributions than for example trimming away the 5\% smallest and 5\% largest observations in the sample.

For a given density the estimators can be compared analytically in the asymptotic case. If $\mathbb{f}$ is the nomal density for instance, the estimators are all asymptotically unbiased and their asymptotic relative efficiencies are given in Table 2. In situation $A, S_{1}$ is asymptotically unbiased. In situation $B$, the asymptotic expectation of $S_{1}$ equals $\sigma(1+8 \varepsilon)^{\frac{1}{2}}$ and that of $S_{2}$ equals $\sigma(1+2 \varepsilon)$. The asymptotic relative efficiency of $\mathrm{S}_{2}$ to $\mathrm{S}_{1}$ is 1.494 for $\varepsilon=0.01$ and 2.354 for $\varepsilon=0.05$ (compared with 0.876 for $\varepsilon=0.0)$.

It is interesting to find out if these - and other - asymptotic results can be transferred to small sample sizes - and if not how the expectations and standard deviations are approaching their asymptotic value. 


\section{METHOD}

We have been using the technique of stochastic simulation (Monte-Carlo Method). The simulation program was written in Simula 67 (Birtwistle (1973)). The stochastic drawings were realized by a pseudo-random generator (Knuth (1969)). The simulations were performed on CD 3300 and CDC-CYBER 74 of the University of 0slo. Simulation results are usually based on 1000 samples for each point in the figures and on 10000 semples for each number in the tables.

During the simulations the parameters were $\xi=0, \sigma=1$. We have worked with $\mathrm{n}$ from 5 to 50 .

To compare the six SD-estimators we have estimated $E_{i}$ by the mean, $\bar{S}_{i}$, and the $S D$ of $S_{i}$ by $S_{1}, S D\left(S_{i}\right)$.

The reason for using $\vec{S}_{i}$ ard $\operatorname{SD}\left(S_{i}\right.$ ) as estimators is that they are traditional, well known measures. Preliminary simulations with the median of the samples as estimator of $E_{i}$ and $S_{5}$ as estimator of $S D$ of $S_{i}$ showed the same tendencies.

As the estimators in many cases are biased we found it sometimes necessary to calculate the mean square error, $E\left(S_{i}-\sigma\right)^{2}$. For small $n$ this is in most cases dominated by var $S_{i}$. We have also studied the empirical distributions of the estimatcrs by histograms.

The estimators $\bar{S}_{i}$ and $S D\left(S_{i}\right), i=1,2, \ldots 6$ will also be compared with their corresponding asymptotic properties. For $i=1,4$ and 5 it is known from statistical textbooks, for example Wilks (1962), that $\sqrt{n}\left(S_{i}-s_{i}\right) / \tau_{i}$ is asymptotic normally distributed, $s_{i}$ and $\tau_{i}$ are given below. For $i=2,3$ and $\sigma \sqrt{n}\left(S_{i}-s_{i}\right) / \tau_{i}$ is more difficult to handle. Bickel and 
Lehmann (1976) prove, however, that if $f$ is symmetric, the estimator of the location parameter may be replaced by the parameter itself in the derivation of the asymptotic properties. Thus it easily follows that $\sqrt{n}\left(s_{i}-s_{i}\right) / \tau_{i}$ is asymptotic normally distributed for $i=2$ and 6 . To prove that this is the case also for $i=3$ we followed Bickel (1965). If $f$ is not symmetric we have deleted $s_{i}$ and $\tau_{i}$ for $i=2,3$ and 6 . We shall refer to $s_{i}$ as the asymptotic expectation and $\tau_{i}^{2}$ as the asymptotic variance of $\sqrt{n} S_{\text {i. }}$ 。

The asymptotic expectations are:

$$
\begin{aligned}
& s_{1}=\sqrt{\operatorname{var} X} \\
& s_{2}=\sqrt{\pi / 2} E|X| \quad \text { (if } f \text { is symmetric) } \\
& s_{3}=\zeta_{0.10} / c_{0.10} \quad \text { (if } f \text { is symmetric) } \\
& s_{4}=\left[F^{-1}(0.841)-F^{-1}(0.159)\right] / 2 \\
& s_{5}=\left[F^{-1}(0.75)-F^{-1}(0.25)\right] / 2 u_{0.75} \\
& s_{6}=H^{-1}(0.5) / u_{0.75} \quad \text { (if } f \text { is symmetric) }
\end{aligned}
$$

where $X$ is a random variable with density $f$ and distribution function $F$. The p-fractile of a distribution, F, is denoted by $F^{-1}(p)$. Further $\zeta_{\alpha}=H_{0}^{-1}(1-\alpha) x d H(x) /(1-\alpha)$ where $H$ is the distribution function of $|X-\mu|$ and $\mu$ is the median of $F$. The asymptotic variances are:

$$
\begin{aligned}
\tau_{1}^{2} & =\left(\tau^{2} / 4\right) \cdot\left(\lambda_{4} / \tau^{4}-1\right) \\
\tau_{2}^{2} & =(\pi / 2) \operatorname{var}|X| \quad(\text { if } f \text { is symmetric) } \\
\tau_{3}^{2} & =\left(\sigma_{\alpha}^{2}-\zeta_{\alpha}^{2}+\alpha\left(H^{-1}(1-\alpha)-\zeta_{\alpha}\right)^{2}\right) /(1-\alpha) c_{\alpha}^{2}, \alpha=0.10 \text { (if } f \text { is symmetric) } \\
\tau_{4}^{2} & =V_{0.841} / 4 \\
\tau_{5}^{2} & =V_{0.75} / 4 \cdot u_{0.75}^{2} \\
\tau_{6}^{2} & =\left[2 \cdot u_{0.75} \cdot h\left(H^{-1}(0.5)\right)\right]^{-2} \text { (if } f \text { is symmetric), }
\end{aligned}
$$


where $\tau^{2}=\operatorname{var} X, \lambda_{4}=E(X-E X)^{4}, \sigma_{\alpha}^{2}=\int_{0}^{H-1}(1-\alpha) x^{2} d H(x) /(1-\alpha)$ and finally

$$
V_{p}=p(1-p) / f_{p}^{2}-2(1-p)^{2} / f_{p} f_{1-p}+p(1-p) / f_{1-p}^{2}
$$

where $f_{p}=f\left(F^{-1}(p)\right)$.

If $F$ is symmetric, $S_{5}$ and $S_{6}$ will have the same asymptotic properties. In this case the expressions for the asymptotic expectations and variances of $S_{4}$ and $S_{5}$ can be reduced.

We have also calculated some rough estimates of the variance of $S D\left(S_{i}\right)$. If $m$ is the number of simulated samples, the approximate variance is $\left(\tau^{2} / 4 m\right) \cdot\left(\lambda_{4} / \tau^{4}-1\right)$ where $\tau^{2}$ and $\lambda_{4}$ are the variance and fourth central moment of $S_{i}$. If the distribution of $S_{i}$ is normal, $\lambda_{4}=3 \tau^{4}$, and the approximate variance is $\tau^{2} / 2 \mathrm{~m}$. Now, repiacing $\tau^{2}$ by an estimate and $m$ by 10000 , we obtain an estimate for the variance of $S D\left(S_{i}\right)$. These estimates vary with $s_{i}, i=1, \ldots, 6$, with the density $f$ and with $n$. After some calculations we found it reasonable to give the estimate $S D\left(S_{i}\right)$ with three decimals, the third decimal being more uncertain the smaller $\mathrm{n}$ is.

The variance of $S_{i}$ being $\tau^{2} / m$, we also give $\bar{S}_{i}$ with three decimals. 


\section{Results}

In the normal case we show in Figure 3 the empirical distributions of $S_{i}, i=1, \ldots, 6$ for $n=20$. The estimators are constructed to be unbiased in this case. The estimated SD's, $\mathrm{SD}\left(\mathrm{S}_{i}\right)$, are given in Table 3 for $\mathrm{n}=5,10,20$ and 50 , they may be compared with $\tau_{i} / \sqrt{n}$ also given in Table 3 which is the approximate $S D$ of $S_{i}$ for large $n$. To get another idea of the accuracy of the simulations, the exact $S D$ of $S_{1}$ for $n=10$ is 0.239 and that of $S_{2}$ is 0.250 and the exact $S D$ of $S_{1}$ for $\mathrm{n}=20$ is 0.163 and that of $S_{2}$ is 0.173 .

We now turn to situation A and choose $f$ as the density of $a W+b$ where $W$ is $x^{2}$-distributed with 6 degrees of freedom (see section 2 case (ii)). In Table 4 the estimated expectations of $S_{i}$ for $n=5,10,20$ and 50 are given. They may be compared to the asymptotic expectation $s_{i}$ given in the last line. The observed biasedness is small compared to $S D\left(S_{i}\right)$ for $n=5,10,20$ and 50 given in Table 5, except for $S_{3}$ and perhaps $S_{6} \cdot S_{2}$ and $\mathrm{S}_{3}$ are the estimators with smallest $S D$. Including the biasedness, however, $S_{2}$ comes out most favourable in this range of $n$.

As the number of degrees of freedom is increasing we are approaching the normal situation and the expectations will tend to 1. The relationship between the SD's will change and $S_{1}$ will become the better one, see Figure $4(a)$ and $(b)$ for $n=20$.

In situation A we have also studied expectation and SD of $S_{i}$ when $f$ is the density of $a V+b$ where $V$ is $t$-distributed (see Section 2 case (i)). The simulated results are given in Figure $5(\mathrm{a})$ and $(\mathrm{b})$ for $n=20$. We also here obtain that the estimators are biased, but that $S_{1}$ behaves better than the other in this respect. $\operatorname{SD}\left(S_{1}\right)$ is, however, larger than $\left.S S_{2}\right)$ 
and $\mathrm{SD}\left(\mathrm{S}_{3}\right)$ and the mean square error of $\mathrm{S}_{2}$ is smaller than the mean square error of $S_{1}$ for $n=20$ and number of degrees of freedom 10 or less.

In situation $A$ we have also considered the case with tails slightly lighter than the normal distribution $(f(x)=$ $(1-\varepsilon) \varphi(x)+\varepsilon u(x), \varepsilon \in<0,0.10]$, where $u(x)$ is the density of a uniform distribution $(-\sqrt{3},+\sqrt{3}))$. The estimators behaved very similar to the pure normal situation.

In situation $B$ we consider the density $f(x)=0.95 \varphi(x / \sigma)$ $+0.05 \varphi(x / 3 \sigma) / 3 \sigma$ (see Section 2 case (iii)), and we are going to estimate $\sigma$. Figure 6 shows the empirical distribution of $s_{i}, i=1, \ldots, 6$ for $n=20$. It is seen that $s_{1}$ is both more biased and more dispersed than the others.

The estimated expectations of $S_{i}$ for $n=5,10,20$ and 50 are given in Table 6. They may be compared to the asymptotic expectation given in the last line of the table. Note that $E_{i}, i=2, \ldots, 6$ seem to approach $s_{i}$ rather quickly. For $\mathrm{n} \geq 20$ the differences are all less than 0.01. The table shows that the "trimmed" estimators, $s_{i}, i=3, \ldots, 6$, behave well with respect to expectation. In Table 7 we give $\operatorname{SD}\left(S_{i}\right)$, $i=1, \ldots, 6$ compared to $\tau_{i} / \sqrt{n}$ for $n=5,10,20$ and 50 . Also with respect to $S D, S_{1}$ behaves worse than the other estimators.

The biasedness and the SD of the estimators are increasing with increasing $\varepsilon$, as might be expected. This is most pronounced for $S_{1}$, as is seen in Figure $7(a)$. In Figure $7(b)$ it is shown how $\operatorname{SD}\left(S_{1}\right)$ is increasing with $\varepsilon$, while $\operatorname{SD}\left(S_{i}\right)$, $i=2,3$ and 5 of the more robust estimators is not very much influenced of $\varepsilon$. 
In situation $B$ we have also considered the case when the wild observations are uniformly distributed with expectation $\xi$ and SD 30. We obtained similar results.

Having carried out the simulations we found that the periodicity of $E_{i}, i=4,5$ which were removed by using the tabulated values of $k_{i}(n)$ for $n<15$, turned up again in $\operatorname{SD}\left(S_{i}\right)$.

Finally, we shall point out by a couple of examples the importance of knowing whether $E S_{i}$ and $S D$ of $S_{i}$ are approaching their asymptotic values $s_{i}$ and $\tau_{i}$ from above or below.

For instance, if the density $f$ is symmetric $S_{5}$ and $S_{6}$ are asymptotically equivalent, specially $\tau_{5}=\tau_{6}$. For $f(x)=\varphi(x)$ or $f(x)=0.95 \varphi(x)+0.05 \varphi(x / 3) / 3$, however, $\sqrt{n} \operatorname{SD}\left(S_{5}\right)$ and $\sqrt{n} \operatorname{SD}\left(S_{6}\right)$ approach their common asymptotic value from opposite sides as can be deduced from Table 3 and Table 7 . In both cases $S_{5}$ is to be preferred.

From Table 6 it is seen that for $f(x)=0.95 \varphi(x)+0.05 \varphi(x / 3) / 3$ $s_{1}$ is larger than the other $s_{i}, i=2, \ldots, 6$. For $n=5$ the difference between $\bar{s}_{1}$ and any $\bar{s}_{i}, i=2, \ldots, 6$ is much smaller. This is because $E_{1}$ is approaching $s_{1}$ from below and for $i=2, \ldots, 6 \mathrm{ES}_{i}$ is approaching $s_{i}$ from above. This is also shown in Figure $8(a)$ for $i=1,2,3$.

At the end of section 3 we pointed out the large asymptotic relative efficiency 2.354 of $S_{2}$ to $S_{1}$ for $f(x)=0.95 \varphi(x)$ $+0.05 \varphi(x / 3) / 3$. For small $n$, say $n \leq 20$, the relative efficiency of $S_{2}$ to $S_{1}$ is much less. This is because $\sqrt{n} \operatorname{SD}\left(S_{1}\right)$ is approaching $\tau_{1}$ from below while $\sqrt{n} \operatorname{SD}\left(S_{2}\right)$ is approaching $\tau_{2}$ from above, se Figure $8(b)$.

Thus the situation for $S_{1}$ is not as bad for finite samples as mignt be thought from only asymptotic considerations. 


\section{Discussion}

The problem of robust SD-estimation is quite difficult compared to estimation of location. We are always running the risk of eliminating important information about the real dispersion in the distribution. For instance, in the case of $t$-distribution, we found that $S_{3}$, which trims away outlayers, caused underestimation of $\sigma$ (Figure $5(\mathrm{a})$ ). To put it another way, we do not know whether possible heavy rails are due to impurities or characterize the underlying distribution. However, we know now that $\mathrm{S}_{1}$ is extremely sensitive against outlayers, and that for reasonable deviations from normality assumptions, more robust estimators should substitute $s_{1}$. In this manner we avoid totally wrong estimates when outlayers are present.

We have also seen that there are great differences between the two fractile-difference estimators considered. Karl Pearson (1920) found that the asymptotic optimal choice of fractile-difference estimator under normality assumptions is obtained by $\left.S=\left(\hat{\mu}_{0.93}-\hat{\mu}_{0.07}\right) / 2 u_{0.93}\right)$. However, robustness of fractile-difference estimators increases with decreasing difference. Earlier simulations with $S$ caused an extremely unrobust estimator (in some situations even worse than $s_{1}$ ). The chosen estimator $\mathrm{S}_{4}$ seems to be a rather reasonable choice. It is quite robust, and has satisfying properties in the normal case. $S_{5}$ is very robust, but perhaps too bad in the normal case.

Even if $S_{5}$ and $S_{6}$ are asymptotically equivalent when $f$ is symmetric, their finite properties are quite different, as they in many situations approach their common asymptotic 
properties from opposite sides. In fact, $S_{5}$ turns out to be better than the asymptotic properties and should therefore be preferred to $\mathrm{S}_{6}$.

The main conclusion of this work is that slightly robust estimators such as $\mathrm{S}_{2}, \mathrm{~S}_{3}$ and $\mathrm{S}_{4}$ should substitute the traditional $\mathrm{S}_{1}$. The actual choice of estimator among these three depends on what we can say about possible deviations from the normality assumption.

A very interesting question concerning SD-estimators is whether a robust estimator can substitute the usual $S_{1}$ in other computations (for instance in students $T=(\bar{x}-\xi) \sqrt{n} / S_{1}$ ). This may be an important method of robustifying statistics and tests. We have performed a few simulations along this line, and it seems to work out very well. Further simulations are necessary and we hope to give these results in a future paper. 


\section{REFERENCES}

1. Andrews, D.F., Bickel, P.J., Hampel, F.R., Huber, P.J., Rogers, W.H., Tukey, J.W. (1972). Robust Estimates of Location. Survey and Advances, Princeton University Press.

2. Bickel, P.J. (1965). On Some Robust Estimates of Location. The Annals of Mathematical Statistics, Vol. 33, 847-858.

3. Bickel, P.J. \& Lehmann, E.L. (1976). Descriptive Statistics for Nonparametric Models. III Dispersion. The Annals of Statistics Vol. 4 No. 6, 1139-1158.

4. Birtwistie, G.M., DahI, O.-J., Myhrhaug, B., Nygaard, K. (1973). Simula Begin. Auerbach, Philadelphia.

5. Knuth, D.E. (1969). The Art of Computer Programming, Vol. 2 . Seminumerical Algorithms. Addison-Wesley, Reading.

6. Pearson, K. (1920). On the Probable Error of Frequency Constants. Biometrika, Vol. 13, 113-132.

7. Wilks, S.S. (1962). Mathematical Statistics. John Wiley \& Sons. New York, London.

Grete U. Fenstad

Department of Mathematics

University of Oslo

P.0.Box 1053, Blindern

Oslo 3, Norway 


\begin{tabular}{|c|c|c|c|c|c|c|c|c|c|c|}
\hline $\mathrm{n}$ & 5 & 6 & 7 & 8 & 9 & 10 & 11 & 12 & 13 & 14 \\
\hline $\mathrm{k}_{4}(\mathrm{n})$ & 1.0340 & 1.0156 & 1.0111 & 1.0162 & 1.0286 & 1.0293 & 1.0202 & 1.0149 & 1.0129 & 1.0136 \\
\hline $\mathrm{k}_{5}(\mathrm{n})$ & 1.0188 & 1.0510 & 1.0279 & 1.0181 & 1.0187 & 1.0281 & 1.0187 & 1.0146 & 1.0149 & 1.0192 \\
\hline
\end{tabular}

TABLE 1 。

The correction factore $k_{i}(n)=\left(\mathrm{ET}_{i}\right)^{-1}, i=4,5$

for $n<15$, computed by using tables of expected

values of order statistics from the normal distribution.

\begin{tabular}{|c|c|c|c|c|c|c|}
\hline$i$ & 1 & 2 & 3 & 4 & 5 & 6 \\
\hline$\tau_{1} / \tau_{i}$ & 1 & 0.876 & 0.674 & 0.541 & 0.368 & 0.368 \\
\hline
\end{tabular}

TABLE 2 .

The asymptotic relative efficiencies of $S_{i}$ to $S_{1}$

when the observations are normally distributed. 


\begin{tabular}{|l|l|l|l|l||l|l|l|l|l|l|l|l|l|}
\hline & $\mathrm{SD}\left(\mathrm{S}_{1}\right)$ & $\tau_{1} / \sqrt{\mathrm{n}}$ & $\mathrm{SD}\left(\mathrm{S}_{2}\right)$ & $\tau_{2} / \sqrt{\mathrm{n}}$ & $\mathrm{SD}\left(\mathrm{S}_{3}\right)$ & $\tau_{3} / \sqrt{\mathrm{n}}$ & $\mathrm{SD}\left(\mathrm{S}_{4}\right)$ & $\tau_{4} / \sqrt{\mathrm{n}}$ & $\mathrm{SD}\left(\mathrm{S}_{5}\right)$ & $\tau_{5} / \sqrt{\mathrm{n}}$ & $\mathrm{SD}\left(\mathrm{S}_{6}\right)$ & $\tau_{6} / \sqrt{\mathrm{n}}$ \\
\hline $\mathrm{n}=5$ & 0.361 & 0.316 & 0.370 & 0.338 & 0.394 & 0.385 & 0.363 & 0.430 & 0.420 & 0.522 & 0.586 & 0.522 \\
$n=10$ & 0.237 & 0.224 & 0.250 & 0.239 & 0.287 & 0.272 & 0.292 & 0.304 & 0.362 & 0.369 & 0.368 & 0.369 \\
$n=20$ & 0.162 & 0.158 & 0.172 & 0.169 & 0.195 & 0.193 & 0.202 & 0.215 & 0.246 & 0.261 & 0.262 & 0.261 \\
$n=50$ & 0.102 & 0.100 & 0.109 & 0.107 & 0.122 & 0.122 & 0.134 & 0.136 & 0.165 & 0.165 & 0.166 & 0.165 \\
\hline
\end{tabular}

TABLE 3 .

The estimated SD of $S_{i}, i=1,2, \ldots, 6$ for different..n, compared with the SD obtained from the asymptotic value. The observations are normally distributed $(0,1)$.

\begin{tabular}{|r|c|c|c|c|c|c|}
\hline $\mathrm{i}$ & 1 & 2 & 3 & 4 & 5 & 6 \\
\hline 5 & 0.972 & 0.972 & 0.946 & 0.960 & 0.956 & 0.909 \\
10 & 0.983 & 0.974 & 0.922 & 0.956 & 0.945 & 0.900 \\
20 & 0.988 & 0.972 & 0.906 & 0.950 & 0.944 & 0.909 \\
50 & 0.996 & 0.974 & 0.894 & 0.951 & 0.943 & 0.913 \\
\hline \hline \multirow{2}{*}{$\mathrm{s}_{i}$} & 1.000 & - & - & 0.944 & 0.939 & - \\
\hline
\end{tabular}

TABLE 4 .

The table gives $\bar{s}_{i}$ for $n=5,10,20$ and 50 . They may be compared with the asymptotic expectation $s_{i}:$ The observations are distributed as $(W-6) / \sqrt{12}$ where $W$ is $x^{2}$-distributed with 6 degrees of freedom. 


\begin{tabular}{|l|l|l||l||l|l|l|l|l|l|}
\hline & $S D\left(S_{1}\right)$ & $\tau_{1} / \sqrt{n}$ & $S D\left(S_{2}\right)$ & $S D\left(S_{3}\right)$ & $S D\left(S_{4}\right)$ & $\tau_{4} / \sqrt{n}$ & $S D\left(S_{5}\right)$ & $\tau_{5} / \sqrt{1}$ & $S D\left(S_{6}\right)$ \\
\hline$n=5$ & 0.433 & 0.447 & 0.434 & 0.416 & 0.414 & 0.459 & 0.453 & 0.529 & 0.558 \\
$n=10$ & 0.304 & 0.316 & 0.295 & 0.289 & 0.318 & 0.325 & 0.372 & 0.374 & 0.353 \\
$n=20$ & 0.217 & 0.224 & 0.204 & 0.196 & 0.218 & 0.230 & 0.253 & 0.265 & 0.252 \\
$n=50$ & 0.142 & 0.141 & 0.129 & 0.121 & 0.143 & 0.145 & 0.170 & 0.167 & 0.162 \\
\hline
\end{tabular}

\section{TABLE 5.}

The estimated SD of $S_{i}, i=1,2, \ldots, 6$ for different $n$, compared with the SD obtained from the asymptotic value. The observations are distributed as $(w-6) / \sqrt{12}$ where $w$ is $x^{2}$-aistributed with 6 degrees of freedom.

\begin{tabular}{|l|c|c|c|c|c|c|}
\hline $\mathrm{n}$ & 1 & 2 & 3 & 4 & 5 & 6 \\
\hline 5 & 1.137 & 1.125 & 1.101 & 1.127 & 1.092 & 1.065 \\
10 & 1.147 & 1.112 & 1.066 & 1.063 & 1.047 & 1.042 \\
20 & 1.153 & 1.098 & 1.040 & 1.043 & 1.038 & 1.032 \\
50 & 1.176 & 1.104 & 1.038 & 1.048 & 1.037 & 1.036 \\
\hline $\mathrm{s}_{i}$ & 1.183 & 1.100 & 1.048 & 1.046 & 1.039 & 1.039 \\
\hline
\end{tabular}

\section{TABIS 6.}

The table gives $\bar{S}_{i}$ for $n=5,10,20$ and 50 . They may be compared with the asymptotic expectation $s_{i}$. The observations have density $f(x)=0.95 \varphi(x)+0.05 \varphi(x / 3) / 3$.

\begin{tabular}{|lll|l|l|l|l|l|l||l|l||l|l|}
\hline & $\mathrm{SD}\left(\mathrm{S}_{1}\right) \tau_{1} / \sqrt{\mathrm{n}}$ & $\mathrm{SD}\left(\mathrm{S}_{2}\right)$ & $\tau_{2} / \sqrt{\mathrm{n}}$ & $\mathrm{SD}\left(\mathrm{S}_{3}\right)$ & $\tau_{3} / \sqrt{\mathrm{n}}$ & $\mathrm{SD}\left(\mathrm{S}_{4}\right)$ & $\tau_{4} / \sqrt{\mathrm{n}}$ & $\mathrm{SD}\left(\mathrm{S}_{5}\right)$ & $\tau_{5} / \sqrt{\mathrm{n}}$ & $\mathrm{SD}\left(\mathrm{S}_{6}\right)$ & $\tau_{6} / \sqrt{\mathrm{n}}$ \\
\hline $\mathrm{n}=5$ & 0.548 & 0.682 & 0.526 & 0.445 & 0.489 & 0.414 & 0.523 & 0.461 & 0.506 & 0.547 & 0.643 & 0.547 \\
$\mathrm{n}=10$ & 0.405 & 0.483 & 0.345 & 0.315 & 0.320 & 0.293 & 0.334 & 0.326 & 0.387 & 0.387 & 0.393 & 0.387 \\
$\mathrm{n}=20$ & 0.304 & 0.341 & 0.233 & 0.222 & 0.212 & 0.207 & 0.222 & 0.231 & 0.257 & 0.274 & 0.273 & 0.274 \\
$\mathrm{n}=50$ & 0.202 & 0.216 & 0.139 & 0.141 & 0.129 & 0.131 & 0.142 & 0.146 & 0.171 & 0.173 & 0.172 & 0.173 \\
\hline
\end{tabular}

TABLE 7.

The estimated SD of $S_{i}, i=1,2, \ldots, 6$ for different $n$, compared with the SD obtained from the asynptotic value. The observations have density $f(x)=0.95 \varphi(x)+0.05 \varphi(x / 3) / 3$. 

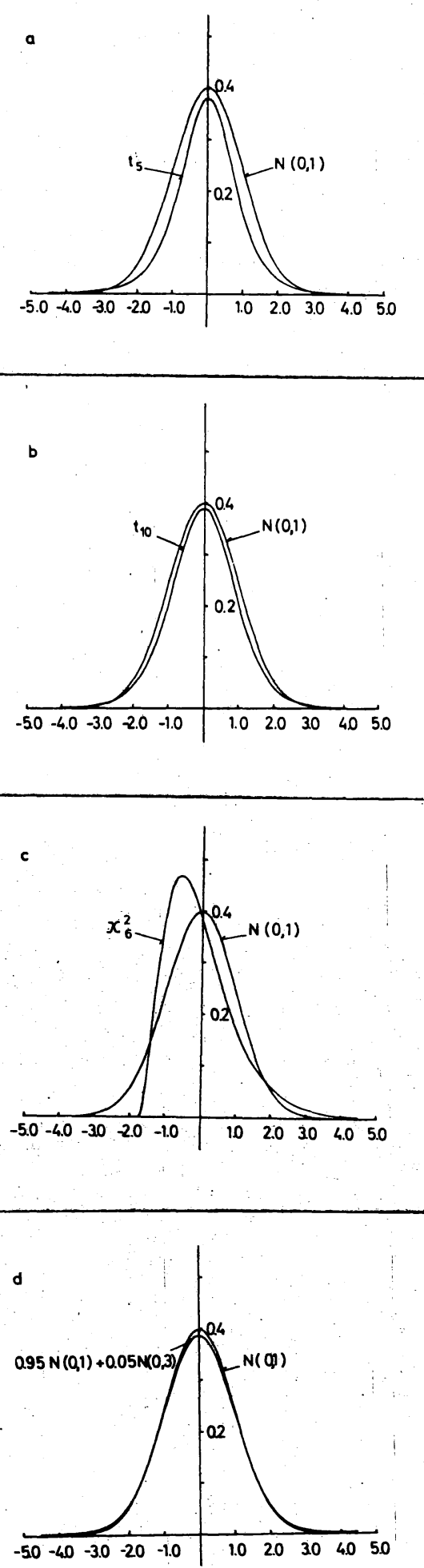

FIGURE 1 .

The normal $(0,1)$ density compared with some of the densities considered in Situations $A$ and $B$ : ( $a$ ) and (b) the densities of the transformed t-distribution with 5 and 10 degrees of freedom, (c) the density of the transformed $x_{6}^{2}$-distribution, and (d) $f(x)=0.95 \varphi(x)+0.05 \varphi(x / 3) / 3$. 

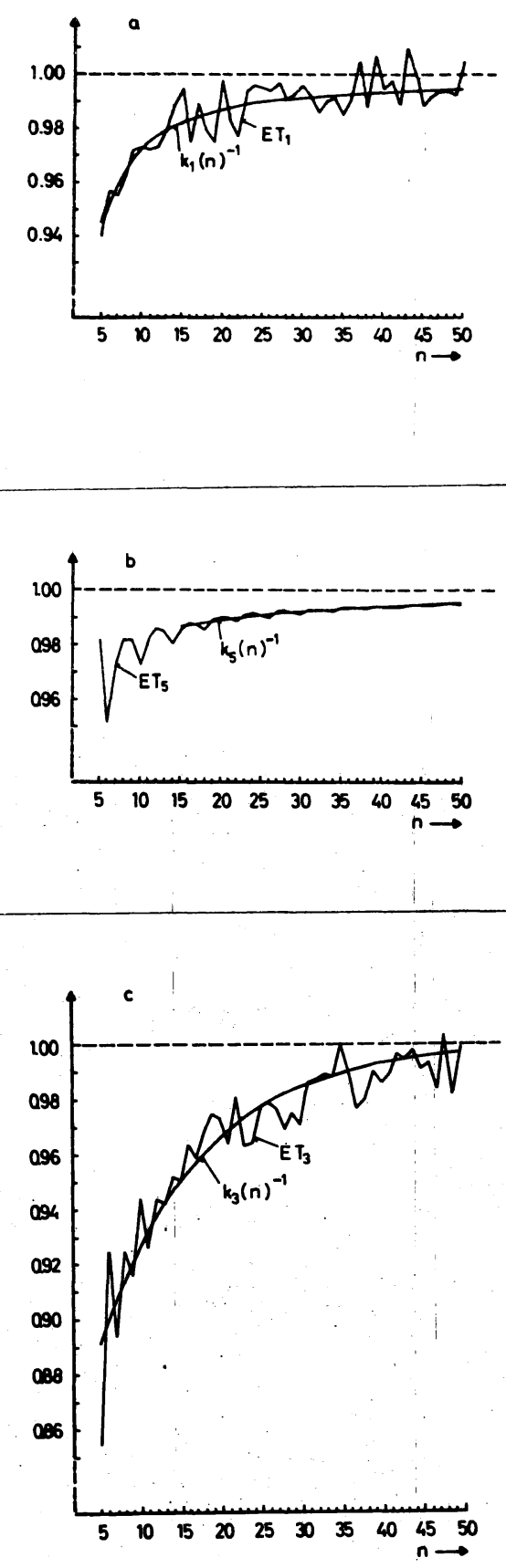

FIGURE 2 .

(a) Simulated estimates of $\mathrm{ET}_{1}$ are compared with $\left[k_{1}(n)\right]^{-1}=\mathrm{ET}_{1}$

(b) $\mathrm{ET}_{5}$ is shown for $5 \leq \mathrm{n} \leq 50$ and compared with $\left[k_{5}(n)\right]^{-1}$ for $n \geq 15$.

(c) Simulated estimates of $\mathrm{ET}_{3}$ are compared with $\left[k_{3}(n)\right]^{-1}$. 

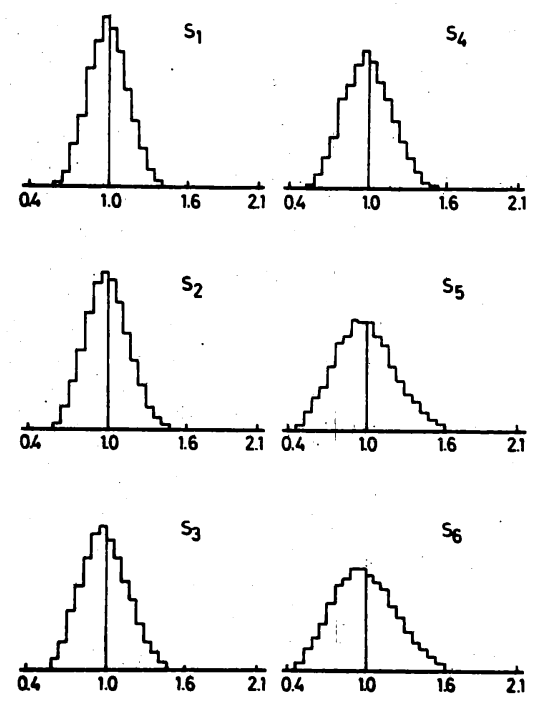

FIGURE 3.

Histograms of $S_{i}, i=1, \ldots, 6$ when $f(x)=\varphi(x)$ based on 10000 samples. 

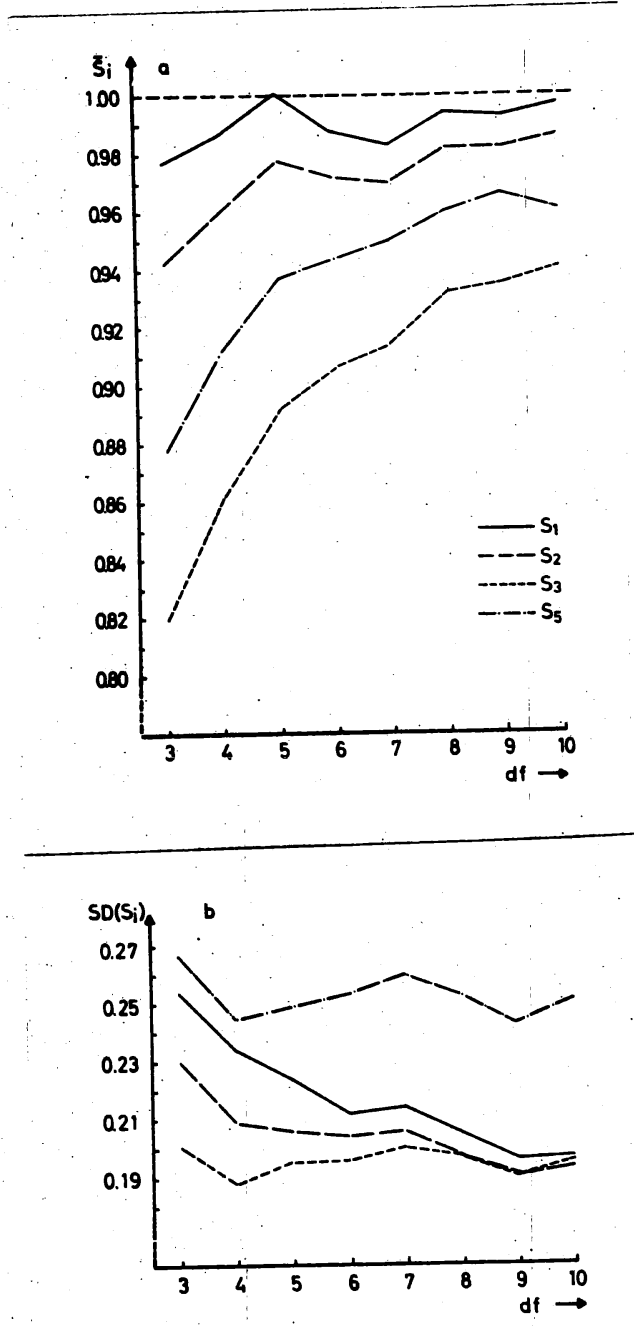

FIGURE 4.

(a) $\bar{S}_{i}$ and (b) $S D\left(S_{i}\right)$ are shown for increasing values of $v$ when the observations are $x_{v}^{2}$-distributed, $n=20$ and $i=1,2,3$ and 5 . 

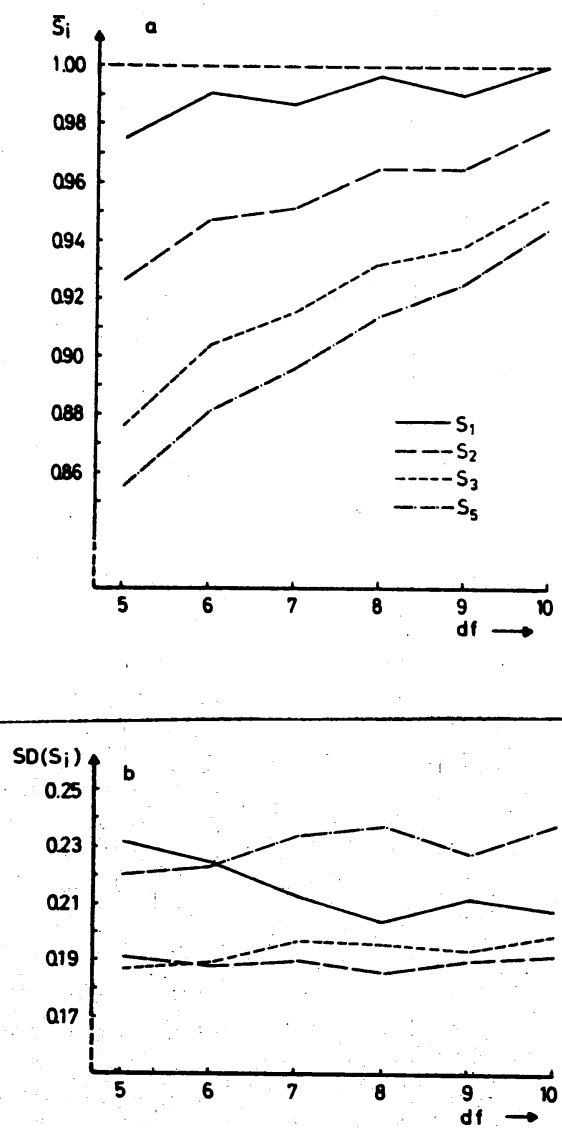

FIGURE 5.

(a) $\bar{S}_{i}$ and (b) $S D\left(S_{i}\right)$ are shown for increasing values of $v$ when the observations are $t$-distributed with $v$ degrees of freedom, $n=20$ and $i=1,2,3$ and 5 . 

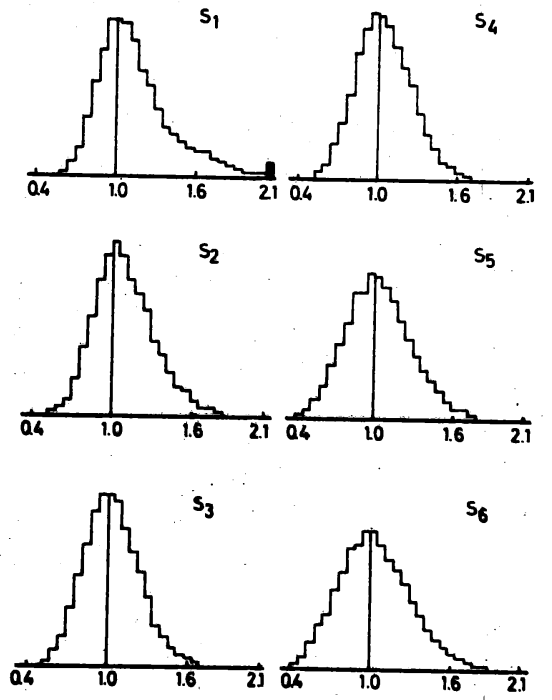

FIGURE 6 .

Histograms of $S_{i}, i=1, \ldots, 6$ when $f(x)=0.95 \varphi(x)+$ $0.05 \varphi(x / 3) / 3$ hased on 10000 samples. 

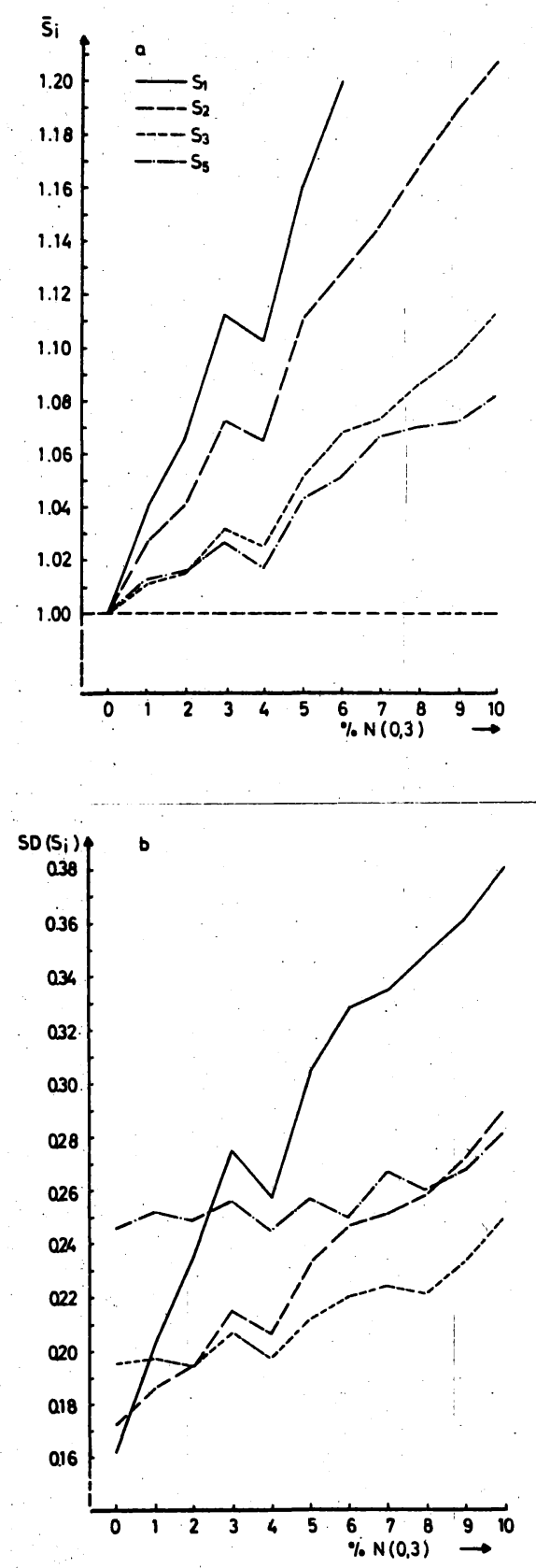

FIGURE 7 .

(a) $\bar{S}_{i}$ and (b) $S D\left(S_{i}\right)$ are shown for increasing values of $\varepsilon$ when the observations have density $f(x)=(1-\varepsilon) \varphi(x)$ $+\varepsilon \varphi(x / 3) / 3, n=20$ and $i=1,2,3$ and 5 . 

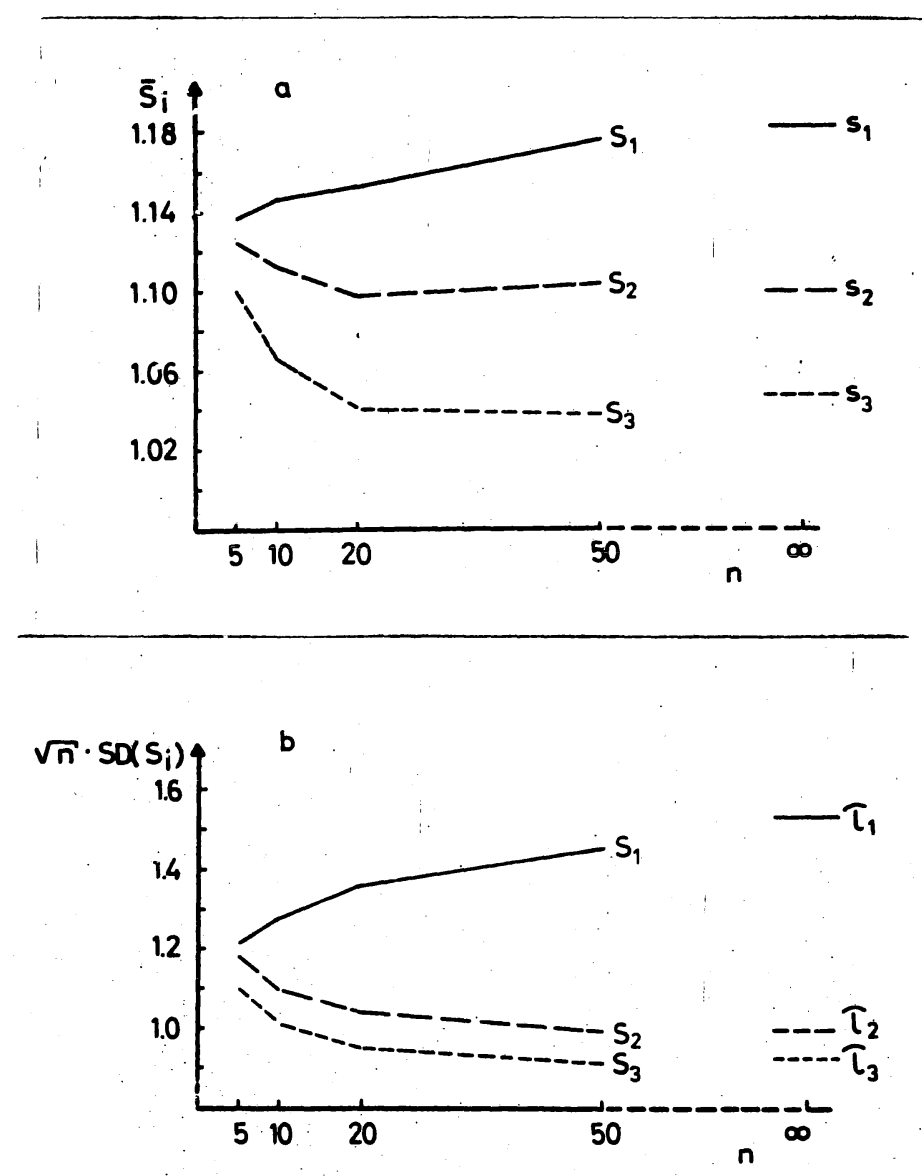

\section{FIGURE 8.}

(a) $\bar{S}_{i}$ and (b) $\sqrt{n} S D\left(S_{i}\right)$ are shown for increasing values of $n$, the observations have density $f(x)=0.95 \varphi(x)$ $+0.05 \varphi(x / 3) / 3, i=1,2,3 . s_{i}$ and $\tau_{i}$ are the asymptotic values. 